\title{
Measuring Engagement at Work: Validation of the Chinese Version of the Utrecht Work Engagement Scale
}

\author{
Ted Chun-tat Fong $\cdot$ Siu-man Ng
}

Published online: 17 June 2011

(C) The Author(s) 2011. This article is published with open access at Springerlink.com

\begin{abstract}
Background Work engagement is a positive work-related state of fulfillment characterized by vigor, dedication, and absorption. Previous studies have operationalized the construct through development of the Utrecht Work Engagement Scale. Apart from the original three-factor 17-item version of the instrument (UWES-17), there exists a nine-item shortened revised version (UWES-9).

Purpose The current study explored the psychometric properties of the Chinese version of the Utrecht Work Engagement Scale in terms of factorial validity, scale reliability, descriptive statistics, and construct validity.

Method A cross-sectional questionnaire survey was conducted in 2009 among 992 workers from over 30 elderly service units in Hong Kong.

Results Confirmatory factor analyses revealed a better fit for the three-factor model of the UWES-9 than the UWES17 and the one-factor model of the UWES-9. The three factors showed acceptable internal consistency and strong correlations with factors in the original versions. Engagement was negatively associated with perceived stress and burnout while positively with age and holistic care climate. Conclusion The UWES-9 demonstrates adequate psychometric properties, supporting its use in future research in the Chinese context.
\end{abstract}

T. C.-t. Fong $(\bowtie) \cdot$ S.-m. Ng

Department of Social Work \& Social Administration,

The University of Hong Kong,

Pokfulam,

Hong Kong, People's Republic of China

e-mail: ttaatt@hku.hk
Keywords Work engagement · Validity · Reliability $\cdot$ Chinese

\section{Introduction}

Despite the traditional focus of psychology on ill-beings, positive psychology, which focuses on the scientific study of human strength and optimal functioning [1], has emerged in the twenty-first century with growing importance. Luthans [2] advocates effective measurement, development, and management of human strengths and psychological capacities that are positively oriented for performance improvement in workplace. Work engagement is defined as a positive, fulfilling, work-related state of mind characterized by vigor, dedication, and absorption [3]. Vigor refers to high levels of energy, mental resilience, and willingness to devote effort in one's work. Dedication is defined as strong involvement in one's work and a sense of significance, pride, challenge, inspiration, and enthusiasm. Absorption refers to being totally concentrated and joyfully immersed in one's work and having difficulty in detaching oneself from work. Engaged workers, who are better connected to the work environment and activities, have a higher level of energy to cope with the demands at work.

The concept of work engagement was operationalized with the Utrecht Work Engagement Scale (UWES) [3]. The UWES is a 17-item self-report instrument (UWES-17) with three dimensions: vigor (six items), dedication (five items), and absorption (six items). A revised 15-item version (UWES-15) was formed by removal of two problematic items [4]. Later, the original authors [5] adopted an iterative process to select the most characteristic 
items from the original scale to form a nine-item short version (UWES-9), with each dimension comprising three items. While previous research has suggested acceptable psychometric properties for the UWES-17 in terms of internal consistency and construct validity $[3,6,7]$, the UWES-9 has been found to exhibit stronger factorial validity $[8,9]$. Because of the apparently strong intercorrelations among the three dimensions of the UWES, Schaufeli and his colleagues [5] have called for using the total composite score as indicator of the overall level of engagement, implying the possibility of the one-factor structure of the UWES.

Work engagement has been linked to various job resources such as social support and procedural justice, positive outcomes such as organizational commitment and job involvement, and negative outcomes such as poorer mental health and turnover intention $[6,10,11]$. Burnout, characterized by exhaustion and cynicism, was negatively linked to work engagement [3]. While vigor and dedication were considered as opposite conditions of exhaustion and cynicism, respectively [6], previous research has revealed relative independence of the two constructs $[5,12]$.

The UWES was widely adopted in international studies with various translated versions such as Italian, Norwegian, Japanese, and Spanish [8, 9, 13]. The robustness and relevance of the construct of work engagement have been demonstrated in different cultures. While researchers have translated the UWES into Chinese and attempted to validate the Chinese version [14], several limitations, namely peculiar deletion of two items before formal validation, abuse of modification indices in confirmatory factor analysis, could be noted in the validation study. Oddly enough, in a study on corporate citizenship, trust, and work engagement, Lin [15] adopted only six out of the original 17 items of the UWES to assess work engagement without any justification or validation. There appears to be a lack of rigorous validation studies of the UWES in the Chinese context. Indeed, having a valid and standardized measurement tool of work engagement in the Chinese context is essential to facilitate a better understanding of work engagement among Chinese workers.

The current study aimed to examine the psychometric properties of the Chinese version of the Utrecht Work Engagement Scale (UWES-C). In particular, the aims were (1) to evaluate the factorial validity, in which we compared the fit of the one-factor model to that of the three-factor model for various versions of the UWES-C, (2) to inspect the scale reliability through Cronbach's alpha and inter-item correlation, (3) to explore the profile of work engagement across demographic subgroups, and (4) to investigate the construct validity through the relationship between work engagement and three validating variables, namely burnout, perceived stress, and holistic care climate.

\section{Methods}

\section{Participants}

This study was part of a larger survey on staff well-being in the elderly service sector of a nongovernmental organization in Hong Kong. A total of 1,067 elderly service workers were invited from over 30 service units to participate in the survey, in which 992 workers joined the survey and completed a selfreport questionnaire. The high response rate $(93 \%)$ could be attributed to the close collaboration between the researchers and the organization together with the anonymous and confidential nature of the data collected. Written informed consent was sought from the participants with reference to relevant ethics codes in Hong Kong.

Among the 992 participants, $83.5 \%$ were women and $16.5 \%$ were men; $68.5 \%$ were married, $22.6 \%$ were single, and $9.0 \%$ were separated or divorced. The majority of the sample (78.7\%) was support staff, with examples such as care workers, workmen, and program workers. Professional staff, such as social workers, nurses, and occupational therapists, accounted for $21.3 \%$ of the sample. The age of the participants ranged from 18 to 62 years (mean $=43.2$, standard deviation $(\mathrm{SD})=10.2$ ), with an average job tenure of 7.9 years $(\mathrm{SD}=6.7)$.

\section{Measures}

Work engagement was measured with the Chinese version of the UWES translated by Zhang and Gan [14]. The original version of the UWES is a 17 -item scale [4] consisting of three dimensions, namely vigor, dedication, and absorption. The items are scored on a sevenpoint Likert scale ranging from 0 ("never") to 6 ("every day").

Burnout was assessed with the Chinese version of the Maslach Burnout Inventory-General Survey [16]. The inventory is a 16-item instrument with three subscales: exhaustion (five items), cynicism (five items), and reduced professional efficacy (six items). The items are scored on a seven-point Likert scale ranging from 0 ("never") to 6 ("always"). In the current sample, confirmatory factor analysis revealed an acceptable fit for the three-factor model $\left(\chi^{2}(101)=416.82, p<.01\right.$, comparative fit index $(\mathrm{CFI})=.92$, root mean square error of approximation $($ RMSEA $)=.06$, standardized root mean square residual $(\mathrm{SRMR})=.06)$. Cronbach's alpha coefficients were .86 for exhaustion, .81 for cynicism, and .77 for reduced professional efficacy, respectively. 
Perceived stress was measured with the Chinese version of the Perceived Stress Scale [17]. The scale is a ten-item instrument that assesses the level of perceived stress during the past month. The two subscales of the instrument are: perceived helplessness (six items) and perceived inefficacy (four items). The items are rated on a five-point Likert scale ranging from 0 ("never") to 4 ("very often"). In the current sample, confirmatory factor analysis revealed an acceptable fit for the two-factor model $\left(\chi^{2}(34)=167.60, p<.01, \mathrm{CFI}=.91\right.$, $\mathrm{RMSEA}=.06, \mathrm{SRMR}=.04)$. Cronbach's alpha coefficients were .76 for perceived helplessness and .66 for perceived inefficacy, respectively.

Holistic care culture was assessed by the Holistic Care Culture Scale, a 13-item self-report instrument that assesses the level of job resources perceived by the worker in the organization [18]. The scale include: caring work environment (five items), social support at work (five items), and sense of mission (three items). The items are scored on a five-point Likert scale ranging from 1 ("strongly disagree") to 5 ("strongly agree"). In the current sample, confirmatory factor analysis revealed an acceptable fit for the three-factor model $\left(\chi^{2}(62)=304.06, p<.01, \mathrm{CFI}=.93, \mathrm{RMSEA}=.06\right.$, $\mathrm{SRMR}=.04)$. Cronbach's alpha coefficients were .81 for caring work environment, .81 for social support at work, and .69 for sense of mission, respectively.

\section{Statistical Analyses}

Structural equation modeling methods were adopted to evaluate the factorial validity of the three (original, revised, and short) versions of the UWES-C. Confirmatory factor analyses were performed with Mplus 5.2 [19] under maximum likelihood robust estimation to examine the goodness of fit of the one-factor and three-factor models. The goodness of fit of the models was evaluated using the following criteria on goodness-of-fit indices: CFI $\geq .90$, Tucker-Lewis index (TLI) $\geq .90$, RMSEA $\leq .08$, and SRMR $\leq .06[20,21]$. Akaike's information criterion (AIC) is a relative measure of parsimony of models, with a lower AIC denoting a more parsimonious model [22]. Nested models were compared using the Satorra-Bentler scaled chisquared difference test [23].

To assess the reliability of the UWES-C, indicators of internal consistency and homogeneity such as Cronbach's alpha coefficients, inter-item correlations, and item-total correlations were scrutinized. Cronbach's alpha coefficients of .70 or higher and item-total correlations of .40 or higher were adopted as the cutoff criteria [24]. Independent $t$ tests and analyses of variance were used to compare the level of the UWES-C across gender, age group, and staff rank using partial eta-squared and Hedge's $g$ as indicators of effect size across subgroups. The construct validity of the UWES-C was evaluated through partial correlations between the UWES-C and the validation variables after control for demographic characteristics. It was anticipated that the UWES-C be positively correlated with holistic care climate while negatively with perceived stress and burnout.

\section{Results}

\section{Factorial Validity of the UWES-C}

Table 1 reports the results of the confirmatory factor analysis of the one-factor and three-factor models of various versions of the UWES-C. Irrespective of the underlying factor structure, both the UWES-17 and UWES-15 fitted the data poorly with CFI and TLI not meeting the criterion of .90 and SRMR exceeding the criterion of .06. For the UWES-9, a marginally acceptable fit was found for the one-factor model to the data (CFI $\geq .90$ and $\mathrm{SRMR}<.06$ but $\mathrm{TLI}<.90$ and RMSEA $>.08$ ). The three-factor model showed an acceptable fit to the data with $\chi^{2}=172.27, d f=24, p<.01, \mathrm{CFI}=.93, \mathrm{TLI}=.90$, $\mathrm{RMSEA}=.08, \mathrm{SRMR}=.05$, and $\mathrm{AIC}=28,401.57$.

In the three-factor model, all of the factor loadings were significant at .01 level with magnitude ranging from .44 to .89 , and the three factors were found to be strongly correlated $(r=.78-.95, p<.01)$. A smaller AIC and a significant result in the Satorra-Bentler scaled chi-squared
Table 1 Results of confirmatory factor analyses of the UWES-C $(N=992)$

$\chi_{M L R}^{2}$ chi-square from maximum likelihood robust estimation, $d f$ degrees of freedom, $C F I$ comparative fit index, TLI Tucker-Lewis index, RMSEA root mean square error of approximation, SRMR standardized root mean residual, $A I C$ Akaike Information Criterion

\begin{tabular}{lcccccccc}
\hline Scale & $\chi_{\text {MLR }}^{2}$ & $d f$ & $p$ & CFI & TLI & RMSEA & SRMR & AIC \\
\hline UWES-17 & & & & & & & & \\
1-factor & 911.19 & 119 & $<.01$ & .83 & .80 & .08 & .07 & $53,973.74$ \\
3-factor & 854.82 & 116 & $<.01$ & .84 & .81 & .08 & .07 & $53,868.17$ \\
UWES-15 & & & & & & & & \\
1-factor & 716.70 & 90 & $<.01$ & .84 & .81 & .08 & .07 & $47,534.00$ \\
3-factor & 700.75 & 87 & $<.01$ & .84 & .81 & .09 & .07 & $47,487.18$ \\
UWES-9 & & & & & & & & \\
1-factor & 229.79 & 27 & $<.01$ & .90 & .87 & .09 & .05 & $28,502.91$ \\
3-factor & 172.27 & 24 & $<.01$ & .93 & .90 & .08 & .05 & $28,401.57$ \\
\hline
\end{tabular}


difference test $\left(\Delta \chi^{2}(3)=49.78, p<.01\right)$ revealed a superior fit for the three-factor model than the one-factor model. As a result, the three-factor model of the UWES- 9 was further scrutinized in terms of descriptive statistics, internal consistency, and construct validity.

\section{Descriptive Statistics and Reliability of the UWES-C}

The descriptive statistics and internal consistency of the UWES-C are displayed in Table 2. Out of the theoretical range of $0-6$, the mean score (standard deviation) was 3.52 (1.08), 3.72 (1.21), 3.89 (1.20), and 2.97 (1.26) for the total score, vigor, dedication, and absorption, respectively. All of the Cronbach's alpha coefficients (.74 for vigor, .77 for dedication, .70 for absorption, and .88 for total scale) were higher than or equal to .70 . All of the nine items were found to be significantly correlated, with inter-item correlations ranging from .19 to .67 . The item-total correlations were significant at .01 level and ranged from .43 to .75 . The correlations between the short and original version of the scales were $.97, .91, .96$, and .92 for total score, vigor, dedication, and absorption, respectively.

\section{Characteristics of the UWES-C Across Demographic Subgroups}

Table 3 reports the descriptive statistics of the UWES-C across demographic subgroups. While male and female workers did not differ significantly in total score $(t=1.67, p=.10)$, dedication ( $t=0.96, p=.34)$, or absorption $(t=1.44, p=.15)$, female workers displayed significantly higher levels of vigor than male workers $(t=2.013$, Hedge's $g=0.18, p<.05)$. A significant difference was found across age groups in total score $(F=21.54, p<.01$, partial eta-squared $=.051)$, vigor $(F=$ $22.29, p<.01$, partial eta-squared $=.052)$, dedication $(F=$ 11.56, $p<.01$, partial eta-squared $=.028)$, and absorption $(F=$ $16.51, p<.01$, partial eta-squared $=.039$ ), respectively. Pairwise comparisons with Bonferroni adjustment showed significantly increasing trends for the UWES-C in which respondents in younger and older age group displayed the lowest and highest score, respectively. Workers in the support rank reported significantly higher levels of total score $(t=$ 2.96 , Hedge's $g=.23, p<.01)$, vigor ( $t=3.98$, Hedge's $g=0.30$, $p<.01)$, and absorption $(t=2.15$, Hedge's $g=.18, p<.05)$ than workers in the professional rank, though they did not differ significantly in the level of dedication $(t=1.66, p=.10)$.

\section{Construct Validity of the UWES-C}

Table 4 displays the correlations between the UWES-C and the validating scales, namely perceived stress, holistic care culture, and burnout, before and after controlling for gender, age, and staff rank. The UWES-C was negatively and weakly correlated with perceived helplessness and perceived inefficacy $(r=-.14$ to $-.25, p<.01)$. Positive associations were found between the UWES-C and holistic care climate at moderate magnitude $(r=.24$ to $.42, p<.01)$. While the UWES-C was negatively associated with exhaustion and cynicism ( $r=-.13$ to $-.37, p<.01)$ at weak to moderate magnitude, negative correlations of stronger magnitude were observed between the UWES-C and reduced professional efficacy $(r=-.36$ to $-.58, p<.01)$.

\section{Discussion}

The UWES is a widely used instrument for measurement of work engagement of the workers. While the Chinese version of the UWES has been available, its psychometric properties have yet to be examined vigorously, thus shedding doubts over its applicability in the Chinese context. The current study attempted to validate the UWES-C by evaluating its psychometric properties in a sample of elderly service workers in Hong Kong. Overall, the UWES-C displayed satisfactory levels of psychometric properties. While confirmatory factor analyses revealed mediocre fits for the original (UWES-17) and revised (UWES-15) versions, the three-factor model of the shortened version (UWES-9) displayed the best model fit with the lowest chi-square statistic, SRMR, and AIC and also the highest CFI and TLI. Such a finding is in line with findings of previous validation studies, in which the UWES-9 exhibited stronger psychometric properties than the UWES-17 [8,9]. For the UWES-9, acceptable internal consistencies were found for the three factors $(\alpha \geq .70)$. Adequate level of scale homogeneity was supported by the significant inter-item correlations among the nine items and substantial item-total correlations $(r \geq .40)$. These results
Table 2 Summary statistics and internal consistency of the UWES-9 $(N=914)$

$S D$ standard deviation, $I Q R$ interquartile range, $\alpha$ Cronbach's alpha, IIR inter-item correlation, ITR item-total correlation

\begin{tabular}{lllllll}
\hline Scale & Mean & SD & IQR & $\alpha$ & IIR range & ITR range \\
\hline Engagement & 3.52 & 1.08 & $2.89-4.22$ & .88 & $.19-.67$ & $.44-.75$ \\
Vigor & 3.72 & 1.21 & $3.00-4.67$ & .74 & $.39-.63$ & $.48-.65$ \\
Dedication & 3.89 & 1.20 & $3.00-4.67$ & .77 & $.46-.62$ & $.53-.65$ \\
Absorption & 2.97 & 1.26 & $2.33-3.67$ & .70 & $.37-.57$ & $.43-.57$ \\
\hline
\end{tabular}


Table 3 Descriptive statistics of the UWES-C across demographic subgroups

\begin{tabular}{|c|c|c|c|c|c|c|c|}
\hline \multirow[b]{2}{*}{ Mean (SD) } & \multicolumn{2}{|l|}{ By gender } & \multicolumn{3}{|l|}{ By age group } & \multicolumn{2}{|l|}{ By staff rank } \\
\hline & $\begin{array}{l}\text { Male } \\
(N=147)\end{array}$ & $\begin{array}{l}\text { Female } \\
(N=752)\end{array}$ & $\begin{array}{l}18-30 \\
(N=134)\end{array}$ & $\begin{array}{l}31-45 \\
(N=279)\end{array}$ & $\begin{array}{l}46-62 \\
(N=396)\end{array}$ & $\begin{array}{l}\text { Support } \\
(N=679)\end{array}$ & $\begin{array}{l}\text { Professional } \\
(N=193)\end{array}$ \\
\hline Engagement & $3.39(1.12)$ & $3.55(1.07)$ & $3.07^{\mathrm{c}}(0.87)$ & $3.46^{\mathrm{b}}(1.00)$ & $3.73^{\mathrm{a}}(1.10)$ & $3.56^{\mathrm{a}}(1.09)$ & $3.32^{\mathrm{b}}(0.97)$ \\
\hline Vigor & $3.54^{\mathrm{b}}(1.27)$ & $3.75^{\mathrm{a}}(1.19)$ & $3.20^{\mathrm{c}}(0.95)$ & $3.65^{\mathrm{b}}(1.09)$ & $3.95^{\mathrm{a}}(1.25)$ & $3.78^{\mathrm{a}}(1.23)$ & $3.43^{\mathrm{b}}(1.05)$ \\
\hline Dedication & $3.81(1.23)$ & $3.91(1.19)$ & $3.51^{\mathrm{c}}(1.03)$ & $3.83^{\mathrm{b}}(1.16)$ & $4.05^{\mathrm{a}}(1.21)$ & $3.92(1.23)$ & 3.77 (1.04) \\
\hline Absorption & $2.82(1.34)$ & $2.99(1.28)$ & $2.49^{\mathrm{c}}(1.02)$ & $2.89^{\mathrm{b}}(1.22)$ & $3.18^{\mathrm{a}}(1.31)$ & $3.00^{\mathrm{a}}(1.28)$ & $2.78^{\mathrm{b}}(1.14)$ \\
\hline
\end{tabular}

Statistically significant difference was found across gender, age groups, and staff rank. Comparison across age groups was done with Bonferroni adjustment

${ }^{\text {a }}$ Subgroup with higher/highest score

${ }^{\mathrm{b}}$ Subgroup with lower score

${ }^{\mathrm{c}}$ Subgroup with lowest score

suggest that the UWES-9 is a reliable measurement scale of work engagement in the Chinese context.

While the superior fit of the three-factor model supports the notion of the three-dimensional nature of work engagement, the three dimensions appear to be highly correlated $(r=.78-.95)$, suggesting the possibility of a higher-order factor. In addition, the total score of the UWES-9 showed good internal consistency $(\alpha=.88)$. This suggests that work engagement may be regarded as a threedimensional as well as a one-dimensional construct. As Schaufeli et al. [5] pointed out, apart from using the scores of the three factors as indicators of the latent engagement construct in structural equation modeling, researchers may opt for the total score as an overall indicator of work engagement. Such an approach avoids problems of multicollinearity that may arise when the three highly related factors are entered in the regression analysis at the same time. Nevertheless, the underlying dimensionality of the UWES-C remains to be elucidated in future research with reference to relevant antecedents and consequences.
After controlling for demographic characteristics, work engagement was found to be significantly associated with the validating variables. Expectedly, work engagement was negatively and moderately associated with burnout. An interesting finding is that work engagement was more strongly correlated with reduced professional efficacy than exhaustion and cynicism. While this finding may imply a close relationship between reduced professional efficacy and engagement, it may be attributed to the pattern of positive wordings for the professional efficacy items [5]. The negative but weak associations between work engagement and perceived stress suggest that workers perceiving higher levels of stress at work were likely to exhibit lower levels of work engagement, albeit to a lesser extent. The fact that work engagement was positively and moderately associated with holistic care climate appears to imply that promoting the culture of holistic care in the workplace could enhance the level of work engagement. Overall, the findings are consistent with previous studies [4, 5, 9, 25], providing support for the construct validity of the UWES-C. However,
Table 4 Correlations between the UWES-C and the validating variables before and after control for gender, age, and staff rank (listwise $N=659$ )

Correlations in brackets denote the partial correlations after control for gender, age, and staff rank $* p<.01$

\begin{tabular}{lcccc}
\hline & Engagement & Vigor & Dedication & Absorption \\
\hline Perceived stress & & & & \\
Perceived helplessness & $-.18^{*}\left(-.14^{*}\right)$ & $-.21^{*}\left(-.19^{*}\right)$ & $-.18^{*}\left(-.16^{*}\right)$ & $-.08(-.04)$ \\
Perceived inefficacy & $-.25^{*}\left(-.25^{*}\right)$ & $-.23^{*}\left(-.24^{*}\right)$ & $-.24^{*}\left(-.24^{*}\right)$ & $-.19^{*}\left(-.18^{*}\right)$ \\
Holistic care climate & & & & \\
Caring work environment & $.37^{*}\left(.37^{*}\right)$ & $.35^{*}\left(.36^{*}\right)$ & $.37^{*}\left(.36^{*}\right)$ & $.26^{*}\left(.26^{*}\right)$ \\
Social support at work & $.37^{*}\left(.38^{*}\right)$ & $.33^{*}\left(.33^{*}\right)$ & $.43^{*}\left(.42^{*}\right)$ & $.23^{*}\left(.24^{*}\right)$ \\
Sense of mission & $.34^{*}\left(.33^{*}\right)$ & $.28^{*}\left(.28^{*}\right)$ & $.35^{*}\left(.32^{*}\right)$ & $.28^{*}\left(.26^{*}\right)$ \\
Burnout & & & & \\
Exhaustion & $-.35^{*}\left(-.30^{*}\right)$ & $.40^{*}\left(-.37^{*}\right)$ & $-.35^{*}\left(-.31^{*}\right)$ & $-.20^{*}\left(-.13^{*}\right)$ \\
Cynicism & $-.35^{*}\left(-.32^{*}\right)$ & $-.39^{*}\left(-.37^{*}\right)$ & $-.37^{*}\left(-.35^{*}\right)$ & $-.18^{*}\left(-.14^{*}\right)$ \\
Reduced professional efficacy & $-.58^{*}\left(-.57^{*}\right)$ & $-.58^{*}\left(-.57^{*}\right)$ & $-.58^{*}\left(-.58^{*}\right)$ & $-.37^{*}\left(-.36^{*}\right)$ \\
\hline
\end{tabular}


the cross-sectional design of the current study implies that no inference can be made on the causal direction of the relationships. Future research could adopt a longitudinal study design to elucidate potential causal relationships.

While female workers appeared to have higher levels of engagement than male workers, the gender contrast was only statistically significant for vigor. Comparison across age groups revealed a consistent trend in which older workers reported significantly higher levels of engagement. The positive association between age and engagement matches with findings from previous research $[5,25]$. An interesting finding is that workers in the support rank showed significantly higher levels of engagement than respondents in the professional rank. This appears to suggest that frontline workers were more engaged to their jobs than management/professional workers. Compared to the normative scores of work engagement in a Spanish sample of 619 workers [3] and a Norwegian sample of 1,266 workers [8], the participants in this study displayed significantly lower levels of vigor $(M=3.72$ vs. 4.12$)$ and dedication $(M=3.89$ vs. 4.44$)$ than the Norwegian sample and significantly lower levels of absorption $(M=2.97$ vs. 3.53) than the Spanish sample. While the considerably lower levels of work engagement in the Chinese context appear to be an interesting finding, discrepancies in composition of study samples in terms of gender, age, and occupation preclude the conclusion of potential crosscultural difference. Further studies should explore potential cross-cultural difference through comparable samples in matched occupational contexts.

\section{Limitations and Conclusion}

There are several limitations in the current study. First, despite the multi-site recruitment and high response rate of study participants, the study sample was based on a specific occupational field of elderly service workers which is primarily composed of female workers. The potential sampling bias implies that the current study results may not be generalized to other occupations. Future studies should scrutinize the UWES-C among workers in male-dominated occupations. Next, the current study did not include any measures on the job demands of the participants. Future research should explore the relationship between the UWES$\mathrm{C}$ and various job demands to elucidate the construct and discriminant validity of the UWES-C. In addition, the selfreport assessment nature of the current study may lead to the problem of common method variance. It is suggested that future studies incorporate objective measures to assess the degree of work engagement.

In summary, the current study provided supportive evidence for the use of the shortened nine-item version of the UWES-C as a satisfactory measure of work engagement in the Chinese context. It is hoped that the validation of the UWES-C will not only promote a better understanding of work engagement in the Chinese context but also inspire international research collaboration on work well-being.

Acknowledgments This study was funded by Caritas Hong Kong. The authors would like to thank the elderly service workers for their participation in this study.

Open Access This article is distributed under the terms of the Creative Commons Attribution Noncommercial License which permits any noncommercial use, distribution, and reproduction in any medium, provided the original author(s) and source are credited.

\section{References}

1. Seligman MEP, Csikszentmihalyi M. Positive psychology: an introduction. Am Psychol. 2000;55:5-14.

2. Luthans F. The need for and meaning of positive organizational behavior. J Organ Behav. 2002;23(6):695-706.

3. Schaufeli WB, Salanova M, Gonzalez-Roma V, Bakker AB. The measurement of engagement and burnout: a two sample confirmatory factor analytic approach. Journal of Happiness Studies. 2002;3:7192.

4. Schaufeli WB, Bakker AB. Utrecht Work Engagement Scale: preliminary manual. Utrecht: Occupational Health Psychology Unit, Utrecht University; 2003.

5. Schaufeli WB, Bakker AB, Salanova M. The measurement of work engagement with a short questionnaire - a cross-national study. Educ Psychol Meas. 2006;66(4):701-16.

6. Schaufeli WB, Bakker AB. Job demands, job resources, and their relationship with burnout and engagement: a multi-sample study. J Organ Behav. 2004;25(3):293-315.

7. van Doornen LJP, Houtveen JH, Langelaan S, Bakker AB, van Rhenen W, Schaufeli WB. Burnout versus work engagement in their effects on 24-hour ambulatory monitored cardiac autonomic function. Stress Heal. 2009;25(4):323-31.

8. Nerstad CGL, Richardsen AM, Martinussen M. Factorial validity of the Utrecht Work Engagement Scale (UWES) across occupational groups in Norway. Scand J Psychol. 2010;51(4):326-33.

9. Shimazu A, Schaufeli WB, Kosugi S, et al. Work engagement in Japan: validation of the Japanese version of the Utrecht Work Engagement Scale. Appl Psychol Int Rev. 2008;57 (3):510-23.

10. Bakker AB, Demerouti E. The job demands-resources model: state of the art. J Manag Psychol. 2007;22(3):309-28.

11. Halbesleben JRB. A meta-analysis of work engagement: relationships with burnout, demands, resources, and consequences. In: Bakker AB, Leiter MP, editors. Work engagement: a handbook of essential theory and research. New York: Psychology Press; 2010. p. 102-17.

12. Hakanen JJ, Bakker AB, Schaufeli WB. Burnout and work engagement among teachers. J Sch Psychol. 2006;43:495-513.

13. Balducci C, Fraccaroli F, Schaufeli WB. Psychometric properties of the Italian version of the Utrecht Work Engagement Scale (UWES-9): a cross-cultural analysis. Eur J Psychol Assess. 2010;26(2):143-9.

14. Zhang YW, Gan YQ. The Chinese version of Utrecht Work Engagement Scale: an examination of reliability and validity. Chin J Clin Psychol. 2005;13(3):268-70. In Chinese.

15. Lin CP. Modeling corporate citizenship, organizational trust, and work engagement based on attachment theory. J Bus Ethics. 2010;94(4):517-31. 
16. Maslach C, Jackson SE, Leiter MP. Maslach burnout inventory manual. 3rd ed. Palo Alto: Consulting Psychologists Press; 1996.

17. Cohen S, Kamarck T, Mermelstein R. A global measure of perceived stress. J Health Soc Behav. 1983;24(4):385-96.

18. Ng SM, Fong TCT, Wang XL. The role of holistic care culture in mitigating burnout and enhancing engagement: a study among elderly service workers in Hong Kong. Aging Ment Health. 2011. doi:10.1080/13607863.2011.556602.

19. Muthen LK, Muthen BO. Mplus user's guide. 5th ed. Los Angeles, CA: Muthen \& Muthen; 1998-2007.

20. Hu L, Bentler PM. Fit indices in covariance structure modeling: sensitivity to underparameterized model misspecification. Psychol Meth. 1998;3:424-53.
21. Schermelleh-Engel K, Moosbrugger H, Muller H. Evaluating the fit of structural equation models: tests of significance and descriptive goodness-of-fit measures. Meth Psychol Res. 2003;8:23-74.

22. Akaike H. A new look at the statistical model identification. IEEE Trans Autom Control. 1974;AC19(6):716-23.

23. Satorra A. Scaled and adjusted restricted tests in multi-sample analysis of moment structures. In: Heijmans RDH, Pollock DSG, Satorra A, editors. Innovations in multivariate statistical analysis. London: Kluwer Academic Publishers; 2000.

24. Nunnaly JC, Bernstein IH. Psychometric theory. 3rd ed. New York: McGraw-Hill; 1994.

25. Schaufeli WB, Taris TW, Van Rhenen W. Workaholism, burnout, and work engagement: three of a kind or three different kinds of employee well-being? Appl Psychol. 2008;57(2):173-203. 